\title{
Explicit Inversion for Two Brownian-Type Matrices
}

\author{
Florendia Valvi ${ }^{1}$, Vassilis Geroyannis ${ }^{2}$ \\ ${ }^{1}$ Department of Mathematics, University of Patras, Patras, Greece \\ ${ }^{2}$ Department of Physics, University of Patras, Patras, Greece \\ Email: fvalvi@upatras.gr, vgeroyan@upatras.gr
}

Received June 22, 2012; revised August 13, 2012; accepted August 20, 2012

\begin{abstract}
We present explicit inverses of two Brownian-type matrices, which are defined as Hadamard products of certain already known matrices. The matrices under consideration are defined by $3 n-1$ parameters and their lower Hessenberg form inverses are expressed analytically in terms of these parameters. Such matrices are useful in the theory of digital signal processing and in testing matrix inversion algorithms.
\end{abstract}

Keywords: Brownian Matrix; Hadamard Product; Hessenberg Matrix; Numerical Complexity; Test Matrix

\section{Introduction}

Brownian matrices are frequently involved in problems concerning "digital signal processing". In particular, Brownian motion is one of the most common linear models used for representing nonstationary signals. The covariance matrix of a discrete-time Brownian motion has, in turn, a very characteristic structure, the so-called "Brownian matrix".

In [1] (Equation (2)) the explicit inverse of a class of matrices $G_{n}=\left[\beta_{i j}\right]$ with elements

$$
\beta_{i j}= \begin{cases}b_{j}, & i \leq j, \\ a_{j}, & i>j .\end{cases}
$$

is given. On the other hand, the analytic expressions of the inverses of two symmetric matrices $K=\left[\kappa_{i j}\right]$ and $N=\left[v_{i j}\right]$, where

$$
\kappa_{i j}=k_{i} \text { and } v_{i j}=k_{j}, i \leq j,
$$

respectively, are presented in [2] (first equation in p. 113, and Equation (1), respectively). The matrix $K$ is a special case of Brownian matrix and $G_{n}$ is a lower Brownian matrix, as they have been defined in [3] (Equation (2.1)). Earlier, in [4] (paragraph following Equation (3.3)) the term "pure Brownian matrix" for the type of the matrix $K$ has introduced. Furthermore, in [5] (discussion concerning Equations (28)-(30)) the so-called "diagonal innovation matrices" (DIM) have been treated, special cases of which are the matrices $K$ and $N$.

In the present paper, we consider two matrices $A_{1}$ and $A_{2}$ defined by

$$
A_{1}=K \circ G_{n} \text { and } A_{2}=N \circ G_{n} \text {, }
$$

where the symbol $\circ$ denotes the Hadamard product. Hence, the matrices have the forms

$$
A_{1}=\left[\begin{array}{cccccc}
k_{1} b_{1} & k_{1} b_{2} & k_{1} b_{3} & \cdots & k_{1} b_{n-1} & k_{1} b_{n} \\
k_{1} a_{1} & k_{2} b_{2} & k_{2} b_{3} & \cdots & k_{2} b_{n-1} & k_{2} b_{n} \\
k_{1} a_{1} & k_{2} a_{2} & k_{3} b_{3} & \cdots & k_{3} b_{n-1} & k_{3} b_{n} \\
\cdots & & & & & \\
k_{1} a_{1} & k_{2} a_{2} & k_{3} a_{3} & \cdots & k_{n-1} b_{n-1} & k_{n-1} b_{n} \\
k_{1} a_{1} & k_{2} a_{2} & k_{3} a_{3} & \cdots & k_{n-1} a_{n-1} & k_{n} b_{n}
\end{array}\right]
$$

and

$$
A_{2}=\left[\begin{array}{cccccc}
k_{1} b_{1} & k_{2} b_{2} & k_{3} b_{3} & \cdots & k_{n-1} b_{n-1} & k_{n} b_{n} \\
k_{2} a_{1} & k_{2} b_{2} & k_{3} b_{3} & \cdots & k_{n-1} b_{n-1} & k_{n} b_{n} \\
k_{3} a_{1} & k_{3} a_{2} & k_{3} b_{3} & \cdots & k_{n-1} b_{n-1} & k_{n} b_{n} \\
\cdots & & & & & \\
k_{n-1} a_{1} & k_{n-1} a_{2} & k_{n-1} a_{3} & \cdots & k_{n-1} b_{n-1} & k_{n} b_{n} \\
k_{n} a_{1} & k_{n} a_{2} & k_{n} a_{3} & \cdots & k_{n} a_{n-1} & k_{n} b_{n}
\end{array}\right] .
$$

Let us now define for a matrix $B=\left[b_{i j}\right]$ the terms "pure upper Brownian matrix" and "pure lower Brownian matrix", for the elements of which the following relations are respectively valid

$$
b_{i, j+1}=b_{i j}, i \leq j \text {, and } b_{i+1, j}=b_{i j}, i \geq j .
$$

The matrix $A_{1}$ (Equation (4)) is a lower Brownian matrix. Furthermore, the matrix $P N P$, where $P=\left[p_{i j}\right]$ is the permutation matrix with elements 


$$
p_{i j}= \begin{cases}1, & i+j=n+1, \\ 0, & \text { otherwise, }\end{cases}
$$

is a pure Brownian matrix and $P G_{n} P$ a pure lower Brownian matrix. Hence, their Hadamard product $(P N P) \circ\left(P G_{n} P\right)$ gives a pure lower Brownian matrix, that is, the matrix $P A_{2} P$.

In the following sections, we deduce in analytic form the inverses and determinants of the matrices $A_{1}$ and $A_{2}$; and we study the numerical complexity on evaluating $A_{1}^{-1}$ and $A_{2}^{-1}$.

\section{The Inverse and Determinant of $A_{1}$}

The inverse of $A_{1}$ is a lower Hessenberg matrix expressed analytically by the $3 n-1$ parameters defining $A_{1}$. In particular, the inverse $A_{1}^{-1}=\left[\alpha_{i j}\right]$ has elements given by the relations

$$
\alpha_{i j}= \begin{cases}\frac{k_{i+1} b_{i-1}-k_{i-1} a_{i-1}}{c_{i-1} c_{i}}, & i=j \neq 1, n, \\ \frac{k_{2}}{k_{1} c_{1},} & i=j=1, \\ \frac{b_{n-1}}{c_{n-1} c_{n}}, & i=j=n, \\ (-1)^{i+j} \frac{d_{j-1} g_{i} \prod_{v=j+1}^{i-1} k_{v} f_{v}}{\prod_{v=j-1}^{i} c_{v}}, & i-j \geq 1, \\ -\frac{1}{c_{i}}, & j-i=1, \\ 0, & j-i>1,\end{cases}
$$

where

$$
\left\{\begin{array}{lll}
c_{i}=k_{i+1} b_{i}-k_{i} a_{i}, & i=1,2, \cdots, n-1, & c_{0}=1, \quad c_{n}=b_{n} \\
d_{i}=k_{i+1} a_{i+1} b_{i}-k_{i} a_{i} b_{i+1}, & i=1,2, \cdots, n-2, & d_{0}=a_{1}, \\
f_{i}=a_{i}-b_{i}, & i=2,3, \cdots, n-1, & \\
g_{i}=k_{i+1}-k_{i}, & i=2,3, \cdots, n-1, & g_{n}=1,
\end{array}\right.
$$

with

$$
\prod_{v=j+1}^{i-1} k_{v} f_{v}=1 \text { if } i=j+1,
$$

and with the obvious assumptions

$$
k_{1} \neq 0 \text { and } c_{i} \neq 0, i=1,2, \cdots, n \text {. }
$$

To prove that the relations (8)-(10) give the inverse matrix $A_{1}^{-1}$, we reduce $A_{1}$ to the identity matrix $I$ by applying a number of elementary row transformations.
Then the product of the corresponding elementary matrices gives the inverse matrix of $A_{1}$. These transformations are defined by the following sequence of row operations.

Operation 1 (applied on $A_{1}$ and on the identity matrix I):

$$
\text { row } i-\frac{k_{i}}{k_{i+1}} \times \operatorname{row}(i+1), i=1,2, \cdots, n-1,
$$

which transforms $A_{1}$ into the lower triangular matrix $C_{1}$ given by

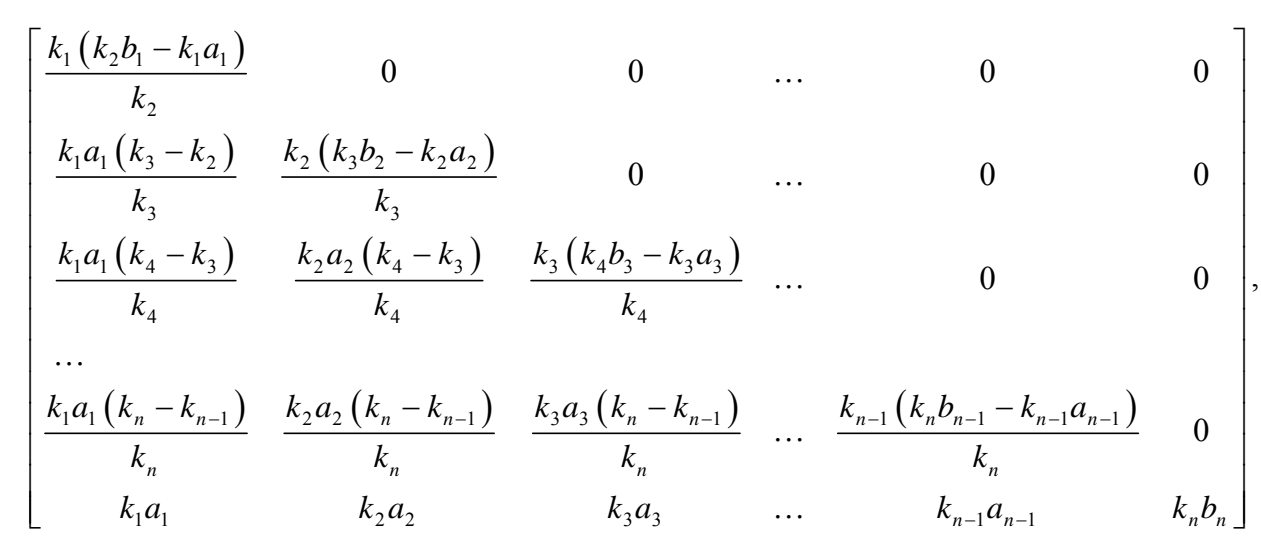

and the identity matrix $I$ into the upper bidiagonal matrix $F_{1}$ with main diagonal

$$
\left(-\frac{k_{1}}{k_{2}},-\frac{k_{2}}{k_{3}}, \cdots,-\frac{k_{n-1}}{k_{n}}\right) .
$$

and upper first diagonal

Operation 2 (applied on $C_{1}$ and $F_{1}$ ): 
row $i-\frac{k_{i} g_{i}}{k_{i+1} g_{i-1}} \times \operatorname{row}(i-1), i=n, n-1, \cdots, 3, k_{n+1}=1$,

which derives a lower bidiagonal matrix $C_{2}$ with main diagonal

$$
\left(\frac{k_{1} c_{1}}{k_{2}}, \frac{k_{2} c_{2}}{k_{3}}, \cdots, \frac{k_{n-1} c_{n-1}}{k_{n}}, k_{n} c_{n}\right)
$$$$
\left[\begin{array}{cccccc}
1 & -\frac{k_{1}}{k_{2}} & 0 & \cdots & 0 & 0 \\
0 & 1 & -\frac{k_{2}}{k_{3}} & \cdots & 0 & 0 \\
0 & -\frac{k_{3} g_{3}}{k_{4} g_{2}} & 1+\frac{k_{2} g_{3}}{k_{4} g_{2}} & \cdots & 0 & 0 \\
\cdots & 0 & 0 & \cdots & 1+\frac{k_{n-2} g_{n-1}}{k_{n} g_{n-2}} & -\frac{k_{n-1}}{k_{n}} \\
0 & 0 & 0 & \cdots & -\frac{k_{n}}{g_{n-1}} & 1+\frac{k_{n-1}}{g_{n-1}}
\end{array}\right]
$$

Operation 3 (applied on $C_{2}$ and $F_{2}$ ):

row $2-\frac{k_{2} a_{1} g_{2}}{k_{3} c_{1}} \times$ row 1 and

row $i-k_{i} \frac{k_{i} g_{i} f_{i-1}}{k_{i+1} g_{i-1} c_{i-1}} \times \operatorname{row}(i-1), i=3,4, \cdots, n$, and lower first diagonal

$$
\left(\frac{k_{1} a_{1} g_{2}}{k_{3}}, \frac{k_{2} k_{3} g_{3} f_{2}}{k_{4} g_{2}}, \cdots, \frac{k_{n-2} k_{n-1} g_{n-1} f_{n-2}}{k_{n} g_{n-2}}, \frac{k_{n-1} k_{n} f_{n-1}}{g_{n-1}}\right) ;
$$

while the matrix $F_{1}$ is transformed into the tridiagonal matrix $F_{2}$ given by

$$
\left[\begin{array}{ccccc}
1 & -\frac{k_{1}}{k_{2}} & \cdots & 0 & 0 \\
-\frac{k_{2} a_{1} g_{2}}{k_{3} c_{0} c_{1}} & \frac{k_{2}\left(k_{3} b_{1}-k_{1} a_{1}\right)}{k_{3} c_{1}} & \cdots & 0 & 0 \\
\frac{k_{3} a_{1} g_{3} k_{2} f_{2}}{k_{4} c_{0} c_{1} c_{2}} & -\frac{k_{3} d_{1} g_{3}}{k_{4} c_{1} c_{2}} & \cdots & 0 & 0 \\
\cdots & \frac{s k_{n-1} d_{1} g_{n-1} k_{3} f_{3} \cdots k_{n-2} f_{n-2}}{k_{n} c_{1} c_{2} \cdots c_{n-2}} & \cdots & \frac{k_{n-1}\left(k_{n} b_{n-2}-k_{n-2} a_{n-2}\right)}{k_{n} c_{n-2}} & -\frac{k_{n-1}}{k_{n}} \\
\frac{s k_{n-1} a_{1} g_{n-1} k_{2} f_{2} \cdots k_{n-2} f_{n-2}}{k_{n} c_{0} c_{1} \cdots c_{n-2}} & \frac{s k_{n} d_{1} g_{n} k_{3} f_{3} \cdots k_{n-1} f_{n-1}}{c_{1} c_{2} \cdots c_{n-1}} & \cdots & -\frac{k_{n} d_{n-2}}{c_{n-2} c_{n-1}} & \frac{k_{n} b_{n-1}}{c_{n-1}}
\end{array}\right]
$$

with the symbol $s$ standing for the quantity $(-1)^{i+j}$.

Operation 4 (applied on $C_{3}$ and $F_{3}$ ):

$$
\frac{k_{i+1}}{k_{i} c_{i}} \times \text { row } i, i=1,2, \cdots, n,
$$

which transforms $C_{3}$ into the identity matrix $I$ and the matrix $F_{3}$ into the inverse $A_{1}^{-1}$.

The determinant of $A_{1}$ takes the form which derives the diagonal matrix

$$
C_{3}=\left\lceil\frac{k_{1} C_{1}}{k_{2}} \frac{k_{2} C_{2}}{k_{3}} \cdots \frac{k_{n-1} C_{n-1}}{k_{n}} \quad k_{n} c_{n}\right\rfloor,
$$

and, respectively, the lower Hessenberg matrix $F_{3}$ given by 
Hessenberg matrix with elements given by the relations

$$
\alpha_{i j}= \begin{cases}\frac{k_{i-1} b_{i-1}-k_{i+1} a_{i-1}}{c_{i-1} c_{i}}, & i=j \neq 1, n, \\ \frac{1}{c_{1},} & i=j=1, \\ \frac{k_{n-1} b_{n-1},}{k_{n} c_{n-1} c_{n}} & i=j=n, \\ (-1)^{i+j} \frac{d_{j-1} g_{i} \prod_{v=j+1}^{i-1} k_{v} f_{v}}{\prod_{v=j-1}^{i} c_{v}}, & i-j \geq 1, \\ -\frac{1}{c_{i}}, & j-i=1, \\ 0, & j-i>1,\end{cases}
$$

where

$$
\begin{cases}c_{i}=k_{i} b_{i}-k_{i+1} a_{i}, & i=1,2, \cdots, n-1, \quad c_{0}=1, \quad c_{n}=b_{n}, \\ d_{i}=k_{i} a_{i+1} b_{i}-k_{i+1} a_{i} b_{i+1}, & i=1,2, \cdots, n-2, d_{0}=a_{1}, \\ f_{i}=a_{i}-b_{i}, & i=2,3, \cdots, n-1, \\ g_{i}=k_{i}-k_{i+1}, & i=2,3, \cdots, n-1, g_{n}=1,\end{cases}
$$

with

$$
\prod_{v=j+1}^{i-1} k_{v} f_{v}=1 \text { if } i=j+1
$$

and with the obvious assumptions

$$
k_{n} \neq 0 \text { and } c_{i} \neq 0, i=1,2, \cdots, n .
$$

In order to prove that the relations (13)-(15) give the inverse matrix $A_{2}^{-1}$, we follow a similar manner to that of Section 2. I):

Operation 1 (applied on $A_{2}$ and on the identity matrix

$$
\text { row } i-\operatorname{row}(i+1), i=1,2, \cdots, n-1,
$$

which transforms $A_{2}$ into the lower triangular matrix $D_{1}$ equal to

$$
\left[\begin{array}{ccccc}
k_{1} b_{1}-k_{2} a_{1} & 0 & \cdots & 0 & 0 \\
a_{1}\left(k_{2}-k_{3}\right) & k_{2} b_{2}-k_{3} a_{2} & \cdots & 0 & 0 \\
\cdots & & & & \\
a_{1}\left(k_{n-1}-k_{n}\right) & a_{2}\left(k_{n-1}-k_{n}\right) & \cdots & k_{n-1} b_{n-1}-k_{n} a_{n-1} & 0 \\
k_{n} a_{1} & k_{n} a_{2} & \cdots & k_{n} a_{n-1} & k_{n} b_{n}
\end{array}\right],
$$

and the identity matrix $I$ into the bidiagonal matrix $L_{1}$ with main diagonal

$$
(1,1, \cdots, 1,1)
$$

and upper first diagonal

$$
(-1,-1, \cdots,-1,-1) \text {. }
$$

Operation 2 (applied on $D_{1}$ and $L_{1}$ ):

$$
\begin{aligned}
& \text { row } n-\frac{k_{n}}{g_{n-1}} \times \text { row }(n-1) \text { and } \\
& \text { row } i-\frac{g_{i}}{g_{i-1}} \times \operatorname{row}(i-1), i=n-1, n-2, \cdots, 3,
\end{aligned}
$$

which derives the lower bidiagonal matrix $D_{2}$ with main diagonal

$$
\left(c_{1}, c_{2}, \cdots, c_{n-1}, k_{n} c_{n}\right)
$$

and lower first diagonal

$$
\left(a_{1} g_{2}, \frac{g_{3} k_{2} f_{2}}{g_{2}}, \cdots, \frac{g_{n-1} k_{n-2} f_{n-2}}{g_{n-2}}, \frac{k_{n} k_{n-1} f_{n-1}}{g_{n-1}}\right) ;
$$

while the matrix $L_{1}$ is transformed into the tridiagonal matrix $L_{2}$ with main diagonal

$$
\left(1,1,1+\frac{g_{3}}{g_{2}}, \cdots, 1+\frac{g_{n-1}}{g_{n-2}}, 1+\frac{k_{n}}{g_{n-1}}\right),
$$

upper first diagonal

$$
(-1,-1, \cdots,-1,-1)
$$

and lower first diagonal

$$
\left(0,-\frac{g_{3}}{g_{2}}, \cdots,-\frac{g_{n-1}}{g_{n-2}},-\frac{k_{n}}{g_{n-1}}\right) .
$$

Operation 3 (applied on $D_{2}$ and $L_{2}$ ):

$$
\begin{aligned}
& \text { row } 2-\frac{a_{1} g_{2}}{c_{1}} \times \text { row } 1, \\
& \text { row } i-\frac{g_{i} k_{i-1} f_{i-1}}{g_{i-1} c_{i-1}} \times \text { row }(i-1), \cdots, \\
& \text { row } n-\frac{k_{n} k_{n-1} f_{n-1}}{g_{n-1} c_{n-1}} \times \text { row }(n-1),
\end{aligned}
$$

with $i=3,4, \cdots, n-1$, which yields the diagonal matrix $D_{3}$,

$$
D_{3}=\left\lceil c_{1} c_{2} \cdots c_{n-1} k_{n} c_{n}\right\rfloor,
$$

and the lower Hessenberg matrix $L_{3}$ equal to 


$$
\left[\begin{array}{ccccc}
1 & -1 & \cdots & 0 & 0 \\
-\frac{a_{1} g_{2}}{c_{0} c_{1}} & \frac{k_{1} b_{1}-k_{3} a_{1}}{c_{1}} & \cdots & 0 & 0 \\
\frac{a_{1} g_{3} k_{2} f_{2}}{c_{0} c_{1} c_{2}} & -\frac{d_{1} g_{3}}{c_{1} c_{2}} & \cdots & 0 & 0 \\
\cdots & \frac{s d_{1} g_{n-1} k_{3} f_{3} \cdots k_{n-2} f_{n-2}}{c_{1} \cdots c_{n-2}} & \cdots & \frac{k_{n-2} b_{n-2}-k_{n} a_{n-2}}{c_{n-2}} & -1 \\
\frac{s a_{1} g_{n-1} k_{2} f_{2} \cdots k_{n-2} f_{n-2}}{c_{0} c_{1} \cdots c_{n-2}} & \frac{s k_{n} d_{1} g_{n} k_{3} f_{3} \cdots k_{n-1} f_{n-1}}{c_{1} c_{2} \cdots c_{n-1}} & \cdots & -\frac{k_{n} d_{n-2} g_{n}}{c_{n-2} c_{n-1}} & \frac{k_{n-1} b_{n-1}}{c_{n-1}}
\end{array}\right]
$$

where the symbol $s$ stands for $(-1)^{i+j}$.

Operation 4 (applied on $D_{3}$ and $L_{3}$ ):

$$
\frac{1}{c_{i}} \times \text { row } i, i=1,2, \cdots, n-1 \text {, and } \frac{1}{k_{n} c_{n}} \times \text { row } n,
$$

which transforms $D_{3}$ into the identity matrix $I$ and $L_{3}$ into the inverse $A_{2}^{-1}$.

The determinant of $A_{2}$ has the form

$$
\begin{aligned}
\operatorname{det}\left(A_{2}\right)= & k_{n} b_{n}\left(k_{1} b_{1}-k_{2} a_{1}\right)\left(k_{2} b_{2}-k_{3} a_{2}\right) \\
& \cdots\left(k_{n-1} b_{n-1}-k_{n} a_{n-1}\right),
\end{aligned}
$$

which shows in turn that the matrix $A_{2}$ is singular if $k_{n}=0$, or, adopting the conventions (14), if $c_{i}=0$ for some $i \in\{1,2, \cdots, n\}$.

\section{Numerical Complexity}

The relations (8) and (13) lead to recurrence formulae, by which the inverses $A_{1}^{-1}$ and $A_{2}^{-1}$, respectively, are computed in $O\left(n^{2}\right)$ multiplications/divisions and $O(n)$ additions/substractions. In fact, the recursive algorithm

$$
\begin{aligned}
& \alpha_{i, i+1}=-1 / c_{i}, i=1,2, \cdots, n-1, \\
& \alpha_{i i}=-\alpha_{i, i+1}+\frac{b_{i-1} g_{i}}{c_{i-1} c_{i}}, \quad i=2,3, \cdots, n-1, \\
& \alpha_{11}=\frac{k_{2}}{k_{1} c_{1}}, \quad \alpha_{n n}=\frac{b_{n-1}}{c_{n-1} c_{n}}, \\
& \alpha_{i, i-1}=-\frac{d_{i-2} g_{i}}{c_{i-2} c_{i-1} c_{i}}, i=2,3, \cdots, n, \\
& \alpha_{i, i-s-1}=-\frac{d_{i-s-2} k_{i-s} f_{i-s}}{d_{i-s-1} c_{i-s-2}} \alpha_{i, i-s}, \\
& i=3,4, \cdots, n, \quad s=1,2, \cdots, i-2,
\end{aligned}
$$

where $c_{i}, d_{i}, f_{i}$, and $g_{i}$ are given by the relation (9), computes $A_{1}^{-1}$ in $5 n^{2} / 2+5 n / 2-6 \mathrm{mult} / \mathrm{div}$ (since the coefficients of $\alpha_{i, i-s}$ depends only on the second subscript) and $5 n-9$ add/sub.
In terms of $j$, the above algorithm takes the form

$$
\begin{aligned}
& \alpha_{j-1, j}=-1 / c_{j-1}, j=2,3, \cdots, n, \\
& \alpha_{j j}=-\alpha_{j, j+1}+\frac{b_{j-1} g_{j}}{c_{j-1} c_{j}}, j=2,3, \cdots, n-1, \\
& \alpha_{11}=\frac{k_{2}}{k_{1} c_{1}}, \alpha_{n n}=\frac{b_{n-1}}{c_{n-1} c_{n}}, \\
& \alpha_{j+1, j}=-\frac{d_{j-1} g_{j+1}}{c_{j-1} c_{j} c_{j+1}}, j=1,2, \cdots, n-1, \\
& \alpha_{j+s+1, j}=-\frac{g_{j+s+1} k_{j+s} f_{j+s}}{g_{j+s} c_{j+s+1}} \alpha_{j+s, j}, \\
& j=1,2, \cdots, n-2, \quad s=1,2, \cdots, n-j-1 .
\end{aligned}
$$

For the computation of $A_{2}^{-1}$ the algorithms (18)-(21) changes only in the estimation of the diagonal elements, for which we have

$$
\begin{aligned}
& \alpha_{i i}=-\alpha_{i, i+1}+\frac{a_{i-1} g_{i}}{c_{i-1} c_{i}}, i=2,3, \cdots, n-1, \\
& \alpha_{11}=-\alpha_{12}, \quad \alpha_{n n}=\frac{k_{n-1} b_{n-1}}{k_{n} c_{n-1} c_{n}},
\end{aligned}
$$

where $c_{i}, d_{i}, f_{i}$, and $g_{i}$ are given by the relation (14). Therefore, considering the relations (9) and (14), it is clear that the number of mult/div and add/sub in computing $A_{2}^{-1}$ is the same with that of $A_{1}^{-1}$.

\section{Concluding Remarks}

The matrices $A_{1}$ and $A_{2}$ represent generalizations of known classes of test matrices. For instance, the test matrices given in [6] (Equations (2.1) and (2.2)) and in [1] (Eq. (2)) belong to the categories presented. Furthermore, by restricting the $a$ 's and $b$ 's to unity, $A_{1}$ and $A_{2}$ reduce to the matrices given in [2]. Also, the matrices in [7] (pp. 41, $42,49)$ are special cases of $A_{1}$ and $A_{2}$. On the other hand, concerning the recursive algorithms given in Section 4, we have performed numerical experiments by assigning 
random values to the parameters of $A_{1}$, and with a variety of the order $n$ from 256 to 1024 . We have found that computing $A_{1}^{-1}$ by the recursive algorithms (18)-(21) is $\sim 100$ times faster than using the LU decomposition when $n=256$ and increases gradually to $\sim 1000$ times faster when $n=1024$.

\section{REFERENCES}

[1] R. J. Herbold, "A Generalization of a Class of Test Matrices," Mathematics of Computation, Vol. 23, 1969, pp. 823-826. doi:10.1090/S0025-5718-1969-0258259-0

[2] F. N. Valvi, "Explicit Presentation of the Inverses of Some Types of Matrices," IMA Journal of Applied Mathematics, Vol. 19, No. 1, 1977, pp. 107-117. doi:10.1093/imamat/19.1.107

[3] M. J. C. Gover and S. Barnett, "Brownian Matrices: Properties and Extensions," International Journal of Sys- tems Science, Vol. 17, No. 2, 1986, pp. 381-386. doi: $10.1080 / 00207728608926813$

[4] B. Picinbono, "Fast Algorithms for Brownian Matrices," IEEE Transactions on Acoustics, Speech and Signal Processing, Vol. 31, No. 2, 1983, pp. 512-514. doi:10.1109/TASSP.1983.1164078

[5] G. Carayannis, N. Kalouptsidis and D. G. Manolakis, "Fast Recursive Algorithms for a Class of Linear Equations," IEEE Transactions on Acoustics, Speech and Signal Processing, Vol. 30, No. 2, 1982, pp. 227-239. doi:10.1109/TASSP.1982.1163876

[6] H. W. Milnes, "A Note Concerning the Properties of a Certain Class of Test Matrices," Mathematics of Computation, Vol. 22, 1968, pp. 827-832. doi:10.1090/S0025-5718-1968-0239743-1

[7] R. T. Gregory and D. L. Karney, "A Collection of Matrices for Testing Computational Algorithms," Wiley-Interscience, London, 1969. 